\title{
Ueber die Entstehung des Schichtstaars und verwandter Staarformen.
}

\author{
Von \\ Dr. Albert Peters, \\ Privatdocenten für Augenheilkunde in Bonn.
}

Hierzu Taf. X, Fig. 1-3.

Trotzdem im Laufe der letzten Jahre wiederholt mikroskopische Untersuchungen von Schichtstaaren angestellt werden konnten, so ist doch die Frage nach der Entstehung dieser interessanten Staarform noch keineswegs gelöst, indem die bisherigen Befunde weder in Bezug auf den mikroskopischen Bau noch auch in Hinsicht anf die Genese eine einheitliche, befriedigende Erklärung ergeben haben. Auch der von mir untersuchte, im Folgenden näher zu schildernde Fall weicht in vieler Hinsicht von den bisher bekannten ab und es dürfte schon aus diesem Grunde eine Mittheilung zu rechtfertigen sein. Gleichzeitig aber scheint mir der Befund geeignet, eine Erklärung für die widersprechenden Resultate der verschiedenen Autoren zu geben.

Otto K., geb. am 30. Juni 1891, wurde am 13. October 1891 in's hiesige Friedrich-Wilhelm-Stift wegen mangelnder häuslicher Pflege aufgenommen. Schon kurz nach der Geburt waren Krampfanfülle bemerkt worden, welche nach der Aufnahme in unverminderter Häufigkeit und Intensität fortdauerten. Der Ernährungszustand war ein schlechter; deutliche Anzeichen 
von Rhachitis waren jedoch nicht wahrzunehmen; eine geringe Verdickung der Epiphysen, besonders an den Vorderarmen könnte hierher gerechnet werden.

Pat. war das einzige Kind und wurde 2 Monate zu früh geboren.

Die Mutter starb am 2. Febr. 1892 an einem Herzfehler. Der Vater, Schriftsetzer, ist gesund.

Die Augen des Kindes wurden zuerst anfangs November untersucht, als die Krankenschwester die Wahrnehmung gemacht hatte, dass bei erweiterter Pupille ein grauer Schein in den Augen zu sehen sei.

Nach Atropineinträuflung fand ich beiderseits einen ziemlich grossen, typischen Schichtstaar. Die Corticalis war vollkommen klar mit Ausnahme einer punktförmigen Trübung am vorderen Pole des rechten Auges. Der Kernäquator hob sich scharf von der Umgebung durch seine intensivere Trübung $a b$, während die übrigen Partieen nur hauchig getrübt erschienen. An einzelnen Stellen waren ganz kleine intensivere Trübungen zu erkennen. Eine zusammenhängende Trübungsschicht fand sich weder im Bereiche der Vorder- noch der Hinterfläche, ebenso konnte eine erhebliche Trübung der hinteren Corticalis nicht bemerkt werden. Es bestand jedoch ein auffallendes Missverhältniss zwischen dem relativ geringen Befund und der bei focaler Beleuchtung deutlich sichtbaren hauchigen Trübung der gesammten Kernmasse einerseits, und der Deutlichkeit, mit welcher der normale Augenhintergrund sich darstellte. Die centralen Theile der Linse liessen so viel Licht durch, dass die Pupille fast roth erschien.

Im April war eine Aenderung des Befundes nur insofern zu konstatiren, als die Trübungen etwas intensiver erschienen.

Der Kräftezustand hob sich wider Erwarten, jedoch traten bald darauf wieder heftigere Krampfanfälle auf, das Allgemcinbefinden wurde schlechter und am 13. Mai 1892 erlag das Kind seinem Leiden, nachdem noch heftiges Erbrechen und Athemnoth hinzagetreten war.

Aus äusseren Gründen konnte eine Section des Kindes nicht vorgenommen werden, jedoch gelang es mir, die Einwilligung des Vaters zur Untersuchung der Augen zu erhalten. Ich enucleirte daher kaum 2 Stunden post mortem beide Augäpfel und härtete sie in Müller'scher Lösung, nachdem der Aequator angeschnitten war. Beide Linsen erwiesen sich als vollkommen durchscheinend. 
Nach 14 Tagen wurden die Bulbi im Aequator halbirt. Die übrigen Augentheile erwiesen sich makroskopiseh als normal, dagegen zeigten beide Linsen Veränderungen im Bereiche des hintern Poles, und zwar Trübungen, welche, in beiden Augen verschieden, durchaus nicht dem klinischen Befunde entsprachen.

Während am rechten Auge fast die ganze Hinterfläche der Linse intensiv weiss getrübt erschien - die Trübung begann überall erst in ca. $1 \mathrm{~mm}$ Entfernung vom Aequator fand sich am linken Auge Folgendes: die Hinterfläche der Linse erscheint zum grössten Theile klar. Ca. $1 \mathrm{~mm}$ rom Aequator entfernt beginnt ein Trübungsring von geringer Breite, welcher nach dem Pole zu 8 radiäre spitze Ausläufer entsendet, welche in einiger Entfernang rom Pole endigen, so dass eine zierliche Rosette ungetrübter Linsensubstanz am Pole frei bleibt. Dadurch wird für den Trübungsring eine wechselnde Breite geschaffen, indem die Ausläufer mit breiter Basis aufsitzen und spitz endigen. Die äquatoriale Zone ist vollkommen klar.

Dieser auffallende Befund, der mit den intra vitam beobachteten Erscheinungen nicht übereinstimmte, veranlasste mich, entgegen meiner ursprünglichen Absicht, die Linsen vom Ciliarkörper abzulösen und für sich allein zu untersuchen, weil ich gespannt war, zu sehen, wie sich die Verhältnisse im Bereiche des vorderen Poles gestaltet hätten. Diese nach einigen Wochen vorgenommene Untersuchung ergab, dass die vorderen Theile der Linse mit feinen punktförmigen Trübungen durchsetzt, im Uebrigen aber ziemlich klar waren. Details im Inneren waren jedoch wegen der intensiven Trübung der Kernmasse und der hinteren Corticalis sonst nicht zu erkennen, ebenso war eine genaue Abgrenzung der Staarschicht nicht mehr möglich.

Beide Linsen wurden nun in Alkohol von allmählich steigender Concentration nachgehärtet und in Celloidin eingebettet.

Bei der Anfertigung der Schnitte mit dem Mikrotom konnte beobachtet werden, dass in beiden Linsen in der Nähe des horizontalen Meridians mit trüber Flüssigkeit und Detritus gefüllte Hohlräume auftraten, welche den später zu schildernden Spaiten entsprachen. Die Untersuchung der aus einer der grössten Spalten mit einer feinen Nadel entnommenen Partikel ergab, dass es sich um feinkörnigen Detritus handelte, der weit eher der Härtungsflüssigkeit als der Linsensubstanz za entstammen schien, da weder Faserreste noch Kugeln oder Tröpfchen gefunden wurden und die Gestalt und Anordnung 
dieser Lücken entschieden auf die Entstehung durch die Härtung hinweist.

Diese Detritusmassen sind nur an einer centralen Stelle der linken Linse so compact, dass sie als Scheibe aus dem Schnitt herausfallen, während im Uebrigen von Anfang an ein noch näher zu schilderndes System von leeren Spalten entstanden war. Im übrigen ist aus den zahlreichen intra vitam vorhandenen Spalten und Lücken auch nicht in einem Schnitte etwas herausgefallen, so dass die auf Rechnung der Härtung zu setzenden Hohlräume mit diesen nicht zu verwechseln sind.

Es empfiehlt sich, beiden Linsen eine gesonderte Beschreibung zu Theil werden zu lassen, da zwar die wesentlichen Punkte übereinstimmen, das makroskopische Aussehen der Schnitte jedoch ein wesentlich versehiedenes ist.

Die Linse des rechten Auges hat einen Durchmesser von $7^{1 / 4} \mathrm{~mm}$ and $4^{1 / 2} \mathrm{~mm}$. Die Dicke erscheint für eine jugendliche Linse sehr gross. Auch weicht die Form insofern $\mathrm{ab}$, als die vordere Flache abnorm gekrümmt erscheint. In der Mitte zwischen vorderem $\mathrm{Pol}$ und Aequator ist je eine leichte Einziehung der Kapsel zu bemerken, ebenso an der hinteren Fläche. Dicht unter der vorderen Kapsel beginnt ein Spaltraum, der nach beiden Seiten spitz zulaufend sich bis zur Stelle jener Einziehung erstreckt. Seine Länge beträgt $3 \mathrm{~mm}$, die grösste Breite am vorderen Pol 1,25 mm. Dieser Spaltraum wird nach innen begrenzt von einer feinen weissen Linie, welche ihn von einer helleren Schicht von wechselnder Breite trennt.

Die Dicke dieser nach beiden Seiten spitz anslaufenden Schicht beträgt in der Mitte ca. $1 \mathrm{~mm}$. Sie bildet ebenso wie eine an der Hinterseite des Kerns befindliche Schicht von gleicher Beschaffenheit eine deutliche Abgrenzung für die Kernsubstanz. Diese hintere Sehicht, die gleichfalls durchseheinend ist, wird von 2 Halbkreisen begrenzt, die einen Zwischenraum von ca. $1 \mathrm{~mm}$ Breite einschliessen; am Kernäquator hört sie beiderseits mit scharfer Grenze auf.

Von diesen beiden durchscheinenden Schichten wird ein eigenthümlich gestalteter Kern eingeschlossen. Derselbe hat einen Durchmesser von $4 \frac{1}{2} \mathrm{~mm}$ und $2 \mathrm{~mm}$. Die Vorderseite erscheint in der Mitte tief eingezogen, die hintere ebenfalls an 2 Stellen, die dem Kernäquator nahe liegen. In diese Einkerbungen senkt sich die helle Schicht hinein, wodurch ihre wechselnde Breite za Stande kommt. Der Kern ist trüber als 
diese Schichten, aber im Ganzen noch durchscheinend. Zwischen Centrum und Hinterfläche des Kerns ist ein schmaler Spalt sichtbar.

Die scharf abgegrenzte Corticalis ist am Aequator bis zu $11 / 2 \mathrm{~mm}$, am hinteren Pol bis zu ca. $3 / 4 \mathrm{~mm}$ breit.

An mehr tangential geführten Schnitten erscheint der Kern mehr und mehr oval, die vordere helle Schicht feblt und ist ersetzt durch ein deutlich getrübtes Band von ca. $1 / 4 \mathrm{~mm}$ Dicke. Hinten ist die helle Sehicht vorhanden and sehr verbreitert. Der zwischen Kern und vorderem Pol gelegene grosse Spaltraum verschwindet allmäblich.

Auf Schnitten, die den Kern nicht mehr getroffen haben, ist in der Mitte eine helle Masse sichtbar, die von einer leicht getrübten Zone eingefasst wird.

Die zweite Linse zeigt ein ganz anderes Bild. Während die Grösse ungefähr der der ersten entspricht $\left(7^{1} / 2: 4^{3} / 4 \mathrm{~mm}\right)$, fehlen hier die leichten Einziehungen der hinteren Kapsel in der Nähe des Aequators, die vordere Fläche ist durch Einbettung an den Rand des Gefässes nur mit einer dünnen Schicht Celloidin bedeckt und leicht abgeflacht.

Dem vorderen Pole entsprechend, zeigt die ziemlich schmale Corticalis einige concentrische schmale Spalten. An diese grenzt eine helle, durchscheinende Schicht von $0,8 \mathrm{~mm}$ Breite, die der oben geschilderten entspricht. Sie erstreckt sich symmetriseh nach beiden Seiten und wird begrenzt von einer getrübten Masse, die in der Nähe des Kernäquators aus der Corticalis vorspringt. Daran schliesst sich auf jeder Seite ein kleiner Spaltraum an und dann wieder die helle Schicht, die nach hinten von wechselnder Breite ist, an einzelnen Stellen bis $\mathrm{zu} 0,8 \mathrm{~mm}$.

Der von diesen Sehichten eingerahmte Kern ist grösser als der in der anderen Linse befindliche. Seine Durchmesser betragen $2^{1 / 2}$ und $5 \mathrm{~mm}$. Die Vorderseite des Kerns ist an verschiedenen Stellen leicht eingekerbt; an der hinteren dem Pole entsprechend etwas tiefer. Der Kern erscheint hier mehr getrübt und ist ron einzelnen grösseren Spalten durchsetzt, welche auf einzelnen Schnitten einen grossen Halbkreis einnehmen, auf anderen in mehrere kleinere zerfallen. Im Centrum ist ein grosser ovaler Spalt sichtbar, aus welchem jene oben beschriebenen Detritusmassen herausgefallen sind. Die Umgebung dieses Spaltes erscheint etwas mehr getrübt als der äbrige Kern. Die Corticalis ist an beiden Polen sehr ver-

*: Graefe' Archiv fir Ophthalmologie. XXXIX. 1. 
schmälert, nach dem Aequator wird sie breiter und ist beiderseits bis zu 1,25 mm dick.

Die hintere Corticalis erscheint in dieser Linse etwas getrübt. Man sieht ca. $1 \mathrm{~mm}$ vom Aequator entfernt eine leichte Abflachung der hinteren Linsenfläche und hier beginnt ein ausgedehnter Spaltraum, dessen Inhalt nicht genau zu bestim men ist, sich von der Corticalis jedoch unterscheidet.

Auf mehr tangentialen Schnitten fehlt zunächst der centrale Spalt im Kern; der Kern selbst wird nach hinten von der hellen Schicht, nach vorne von einer feinen Trübungszone begrenzt. Nach Verschwinden des Kerns treten im Centrum Trübungen auf, die mit helleren Partieen abwechseln.

Die mikroskopische Untersuchung hatte folgendes Ergebniss.

Die Kapsel ist in beiden Linsen überall von ganz normaler Beschaffenheit. Das Epithel ist ebenfalls normal mit Ausnahme einer kleinen Stelle im Bereiche des vorderen Poles der rechten Linse. Hier findet sich eine auf wenige Schnitte beschränkte Verdickung der Epithelschicht, welche die Kapsel an dieser Stelle etwas nach aussen vorgetrieben hat.

Die Kerne sind gut färbbar und von normaler Grösse.

Die makroskopisch sichtbaren Spalten sind an einzelnen Stellen durchsetzt von Faserbündeln, deren Contouren nicht ganz glatt sind. Einzelne Bündel sind in der Mitte scharf abgerissen und es haben sich die Enden weit von einander entfernt. Detritus oder Kugeln haften den Fasern nicht an. Es kann keinem $Z$ weifel unterliegen, dass diese Spaltenbildung und das Zerreissen einzelner Faserbündel zum Theil wenigstens der Härtung zuzuschreiben sind. Nach Gerinnung der eiweisshaltigen Substanz trat eine Schrumpfung ein, welche in Verbindung mit der noch zu schildernden Schrumpfung des Kerns die Festigkeit und den Zusammenhang der Faserbündel und -Schicht überwog und so zur nachträglichen Spaltbildung fübrte. Auch sind zweifellos beim Schneiden der Linsen einzelne Spalträume vergrössert, einzelne Schichten zerrissen worden, da es sich nicht um eine gleichmässig harte Masse handelte. Auch der Umstand, dass sich die Spalten ofters gerade dort gebildet haben, wo zwei verschiedene Schichten aneinander stossen, spricht für postmortale Entstehung, z. B. zwischen Corticalis und der hellen Zwischenschicht; ebenso der, dass die Spalten vollkommen leer sind. Die Summe aller vorhandenen Spalträume ist in beiden Linsen gleich, $d . h$. es ist gleichviel Linsensubstanz und gleichviel an Spalträumen vorhanden. 
Die Anordnung der Spalten ist in beiden Linsen verschieden. Während in der rechten Linse im Wesentlichen sich nur ein Spalt befindet und zwar im Bereiche der vorderen Corticalis, ist in der anderen hauptsächlich der Kern betheiligt.

Diese Spalten erklären auch das grosse Volumen der Linsen, welche ohne dieselben viel flacher erscheinen müssten.

Wir kämen nun zu den Veränderungen in der Anordnung und dem Bau der Linsenelemente selbst.

Zunächst ist hervorzuheben, dass der Kern in hohem Grade verändert ist. Die Peripherie zeigt die oben beschriebenen Einkerbungen, welche mehr als alles Andere für eine intensive Schrumpfung sprechen. Ibnen entspricht die Anordnung der benachbarten Fasern, welche dadurch leicht gebogen erscheinen.

Der ganze Kern ist durchsetzt mit kleinen Tröpfchen von ziemlich gleicher Grösse. Sie besitzen einen DurchschnittsDurchmesser von $0,2 \mu$, sind rundlich und haben ein etwas böheres Brechungsvermögen als die Umgebung. Sie sind an einzelnen Stellen derartig angehäuft, dass keine Fasern mehr sichtbar sind. An anderen Stellen sind sie spärlicher und sie scheinen hier zwischen den Fasern zu liegen. Von einer Lagerung innerhalb der Fasern habe ich mich nirgends überzeugen können. An einzelnen Punkten der Peripherie sind diese Tröpfchen dichter angehänft; eine Regelmässigkeit dieser Anordnung besteht jedoch nicht, insbesondere ist keine bandartige Anordnung zu erkennen. Das Ausselren der Kernsubstanz ist im Allgemeinen ein ziemlich gleichmässiges. Wohl davon zu unterscheiden sind eigenthümliche Gebilde, welche nicht nur dem Kern angehören, sondern auch in der homogenen Schicht um den Kern gefunden werden. Sie sind immer in geraden Linien angeordnet und sind deshalb als Kunstproducte, hervorgerufen durch Unregelmässigkeiten der Schneide des Messers aufzufassen. Sie stellen hellglänzende, strichförmige, zackige oder kugelige Massen dar, welche sich am besten bei oberflächlicher Einstellung des Tubus zeigen. Sie sind einzeln durehaus nicht mit den kleinen Tropfen zu verwechseln, können aber an manchen Stellen eine dichtere Anhäufung derselben vortänschen.

Ferner finden sich zwischen den Fasern im Kern unregelmässig zerstrent wirkliche Lücken. Sie sind meist oval oder spindelförmig; ihre Grösse schwankt erheblich, man sieht einzelne bis $\mathrm{zu} 0,06 \mathrm{~mm}$ gross. Immer sind sie bei Weitem grösser als die Tröpfchen. Eine besondere Anordnung ist 
nicht zu erkennen. Einmal enthält die Peripherie mehr Lücken als das Centrum; ein anderes Mal ist das Umgekehrte der Fall. Der Inhalt der Lücken ist meistens homogen und färbt sich nicht mit Hämatoxylin. In einzelnen, besonders den spindelförmigen, erscheint er mehr gekörnt, in anderen ist ein circulärer Spalt um den Inhalt wahrzunehmen. In diesen Fällen erscheint derselbe von stärkerem Lichtbrechungsvermögen zu sein.

Die Abgrenzung des Kerns ist eine ziemlich scharfe. Hin und wieder treten in der Grenzschicht ganz feine schmale Spalten auf, deren Inhalt homogen und von gleichem Lichtbrechungsvermögen mit dem der Lücken ist. An einzelnen Stellen der Kernperipherie sind die Lücken in eigenthümlicher Weise angeordnet. Es entsteht dadurch ein Bild, welches Lawford ${ }^{1}$ ) treffend mit einem zusammengedrehten Tan vergleicht. Der Oberfläche sind stellenweise kleinere oder grössere Kugeln und feiner Detritus anfgelagert, besonders in den durch die Einkerbungen gebildeten Vertiefungen.

Ueberraschend ist nun, dass dieser so stark veränderte Kern nirgends mit normaler Corticalis im Zusammenhang steht. Linsenfasern sind überhaupt nur an einem Theil der Kernperipherie vorhanden and diese sind ebenfalls durchaus verändert. Betrachten wir zunächst die rechte Linse. Am Aequator des Kerns findet sich eine Schicht von wechselnder Breite, in welcher die Linsenfasern unregelmässige Contouren haben. Ihr Inhalt ist feinkörnig, stellenweise hyalin glänzend. Zwischen den Fasern sind in reichlicher Menge Myelinkugeln und kleine Tröpfchen angehäuft. Letztere sehen denen des Kerns ziemlich ähnlich. Ebenso giebt es schmale kleine Lücken, wie wir sie im Kern sehen.

Diese Schicht ist beiderseits von ziemlich gleicher Dicke and gleicher Ausdehnung; sie sitzt dem Kernäquator auf und erstreckt sich nach dem vorderen Pole zu. Auf mehr tangentialen Schnitten treffen die beiden Schichten dem vorderen Pole entsprechend zusammen. Die grösste Breite beträgt hier öfters $1 / 4 \mathrm{~mm}$. Es zeigt sich nun, dass diese Schicht den einzigen Zusammenhang zwischen Kern und Corticalis darstellt, indern der Kern gewissermassen umgeben ist von einem circulären Aufhängeband, welches am Aequator und an den dem Aequator benachbarten Theilen des Kerns, besonders nach vorne hin, inserirt und von der Corticalis ausgeht. An allen Schnit-

1) s. unten. 
ten, auch an solchen, wo durch die Härtung oder durch Schneiden mit dem Mikrotom Spalten eutstanden sind, lässt sich nachweisen, dass wenigstens ein Faserbündel den Zusammenhang vermittelt. Wo es abgerissen ist, passen die Enden zusammen. Im Uebrigen ist der Kern umgeben von einer homogenen Masse, welche keine einzige Linsenfaser enthält. Sie erscheint in demselben Farbenton wie die übrigen Theile und füllt die Einkerbungen des Kerns aus. Ihre Dicke wechselt; sie ist bis zu $1^{1 / 2} \mathrm{~mm}$ stark und nach hinten mächtiger als nach dem vorderen Pole zu. Bei stärkeren Vergrösserungen erscheint die Masse von feinsten Streifchen und Körnchen durchsetzt; es ist dies jedoch nicht der Ausdruck für eine Durchsetzung mit festen Massen, sondern es handelt sich um moleculare Veränderungen durch Gerinnung und um Kunstproducte durch die Ungleichmässigkeit der Schneide des Messers. Es kann keinem Zweifel unterliegen, dass wir eine Schicht vor uns haben, die eine geronnene Flüssigkeit darstellt.

Der Uebergang in den Kern ist ein ziemlich scharfer, was am Aequator nicht der Fall ist, wo jene getrübte Faserschicht angrenzt.

Die Corticalis ist im Grossen und Ganzen wenig verändert. Die Veränderungen betreffen sowohl die Fasern selbst als auch die Zwischensubstanz. Erstere sind an den Stellen, wo sie an die den Kernäquator einfassende Trübungsschicht angrenzen, erheblich verdickt, stellenweise zu ganz dicken Kolben ausgewachsen, ein Verhalten, das auf mehr tangentialen Schnitten noch deutlicher hervortritt. An einzelnen Stellen sind Spalten vorhanden, die von einem Streifen Corticalis durchzogen werden. Immer lässt sich nachweisen, dass zwischen Corticalis und der getrübten Schicht einerseits und dieser und dem Kern andererseits intra vitam ein directer fester Zusammenhang bestanden hat.

Ferner zeigt die Corticalis besonders in der Nähe der vorderen Kapsel, aber auch im hinteren Theile, eine Reihe von spindelförmigen oder rundlichen Lücken, die gelegentlich eine erhebliche Grösse erreichen. Der Inhalt besteht aus aneinander gereihten Kugeln, ebenso zeigen die Fasern selbst einen solchen Inhalt. Es sind dies Veränderungen, wie sie o. Becker ${ }^{1}$ ) ausführlich beschrieben hat.

1) Zur Anatomie der gesunden und kranken Linse. Wiesb. 1883. Fig. 11, 12, 13, 14. Tafel III. 
Gelegentlich tritt in den äquatorialen Theilen der Corticalis ein grösserer, mit feinkörniger Masse gefullter Spalt anf.

Die postmortal entstandene Spaltenbildung hat in der vorderen Hälfte der Linse zur Abreissung eines schmalen Streifens von Corticalis geführt, welcher zwischen den Fasern ganz schmale Lïcken von ähnlicher Beschaffenheit enthält, wie man sie auch in der Trübungsschicht am Kernäquator findet und wie sie Beselin ${ }^{1}$ ) auf $\mathrm{S}$. 76 beschreibt. Die hintere Kapsel steht nicht tuberall mit der Corticalis in Verbindung. Sie ist beiderseits zwischen Aequator und Pol abgelöst. Diese Ablösung beginnt etwas vom Aequator entfernt mit einer kleinen Einkerbung der Kapsel and endigt in einiger Entfernung vom Pol. Der so entstandene Spalt ist an der breitesten Stelle $0,2 \mathrm{~mm}$ dick und ist mit derselben homogenen Masse gefüllt, die wir oben kennen gelernt haben. Am Rande ist die Corticalis mit einer Schicht dicker, ovaler Myelinkugeln besetzt. Die beiden Einziehungen der Corticalis nach innen entsprechen genau den beiden tiefen Finkerbungen des Kerns.

Die linke Linse zeigt einige Abweichungen. Hier hat die postmortale Spaltbildung im Wesentlichen die Kernsubstanz betroffen. Der Kern selbst zeigt genau dieselben Veränderungen wie der andere, nur erscheint die Durchsetzung mit kleinen Tröpfehen stellenweise noch erheblicher.

Getrennt ist der Kern von der Corticalis gleichfalls durch äquatoriale Trübungszonen und einen grossen vorderen und hinteren Spaltraum, der mit derselben Flüssigkeit gefüllt ist, wie in der rechten Linse.

Der Spalt ist vorne beträchtlich breiter als hinten und auch breiter als in der anderen Linse. Die Einkerbungen des Kerns sind auch hier vorhanden und sie werden auch hier von jener Flüssigkeit ausgefüllt.

Die äquatoriale Trübungszone ist fast uberall durch postmortal entstandene Spalten vom Kern losgelöst. Es lässt sich jedoch deutlich erkennen, dass intra vitam ein fester Zusammenhang bestanden hat, indem am Kern noch Reste dieser Schicht haften, welche genau zu den gegenüberliegenden Theilen passen.

Auch hier sind die Fasern unregelmässig contourirt, nach der Corticalis zu kolbig verdickt und varikös angeschwollen, der Inhalt einzelner Fasern feinkörnig und glänzend. Ebenso

1) Archiv für Augenheilkunde. Bd. XVIII. 
finden sich hier zwischen den Fasern grössere Myelinkugeln und kleinere Tröpfchen wie im Kern. Diese so veränderte Masse sitzt hier nicht gewissermassen als Verbreiterung dem Kern auf, wie in der rechten Linse, sondern es sind hier Spalten aufgetreten, welche mit jener homogenen Masse gefüllt sind und an einzelnen Stellen von einem schmalen Band von Linsenfasern durchzogen werden, welches mit dem Kernäquator einerseits und der Corticalis andererseits in Verbindung steht. An anderen Stellen ist die Trübungsschicht verbreitert. Ein Theil der Schicht ist dem Kern aufgelagert und dadurch kommt der Eindruck zu Stande, als ob hier die Tropfen and Lücken stärker angehäuft wären. Wenn auch die Grenze des Kerns keine deutliche ist, so lässt sich doch leicht nachweisen, dass diese getrübte Schicht die Fortsetzung abgerissener Faserbündel ist, welche ihrerseits mit der Corticalis in Verbindung standen.

Im Gegensatz $\mathfrak{z}$ der rechten Linse sind hier die Lücken in der Corticalis nur sehr spärlich. Die Anhäufung von Tropfen in den Fasern wird gänzlich vermisst. Grössere Spalten mit feinkörnigem Inhalt treten nur sehr vereinzelt auf.

Auffallend ist die geringe Dicke der hinteren Corticalis. Vom Aequator der Linse und aus der Trübungszone am Aequator des Kerns zieht eine dicke Schicht von Linsenfasern nach dem hinteren Pole zu. Diese Schicht wird plötzlich in der Mitte der Linse auf eine ganz schmale Zone reducirt. Es entsteht dadurch zwischen Corticalis und Flüssigkeitsschicht ein ziemlich breiter Raum, der eine starke Anhäufung von grossen Myelinkugeln zeigt.

Die Ablösung der Corticalis von der hinteren Kapsel findet sich auch hier, jedoch in viel grösserer Ausdehnung. Ganz in der Nähe des Aequators ist die Kapsel leicht eingekerbt, die benachbarten Fasern sind demgemäss etwas gebogen. Von dieser Einkerbung erstreckt sich ein Spaltraum, der am Pole die Dicke von $0,4 \mathrm{~mm}$ erreicht. Gefällt ist der Spalt wiederum mit jener homogenen Masse. An der Corticalis haften auch hier zahlreiche grosse Myelinkugeln.

Die Färbung der Schnitte in Hämatoxylin ergab, dass die gesammte Substanz sich gleichmässig färbte, mit Ausnahme der Lücken in der Kernsubstanz, welche fast ungefärbt blieben.

Kurz zusammengefasst ist beiden Linsen Folgendes gemeinsam. Es bestehen zunächst leere Spalten, welche 
durch die Härtung und zum Theil durch das Schneiden hervorgerufen sind. Der Kern ist in ganz bestimmter Weise verändert. Umgeben ist derselbe am Aequator von einer schon makroskopisch sichtbaren Trübungszone, welche mit dem Kern und der Corticalis in directer Verbindung steht, während der grössere Theil der Vorderfläche und der Hinterfläche des Kerns begrenzt wird von einer Flüssigkeitsschicht, welche einen breiten Spaltraum zwischen Kern und Corticalis ausfüllt. Die Corticalis selbst zeigt an sich nur geringe Veränderungen, ist aber in beiden Linsen mehr oder weniger von der hinteren Kapsel abgelöst. Der so entstandene Spaltraum ist ebenfalls mit Flüssigkeit angefüllt.

Die Deutung dieses Befundes unterliegt keinen Schwierigkeiten. Es besteht eine Schrumpfung des in characteristischer Weise veränderten Kerns, welche zunächst zu einer Ablösung der mit ihm noch in Verbindung stehenden Corticalis von der hinteren Kapsel geführt hat. Später löste sich der Kern in grosser Ausdehnung von der Corticalis selbst los, in der Weise, dass er nur am Aequator mit ihr durch ein schmales Band in Verbindung blieb. Der Kern ist auf diese Weise im Imneren der Linse gleichsam aufgehängt an einem im Aequator circulär verlaufenden Bande getruibter Linsensubstanz, und diese Trübung ist wohl auf eine Zerrung eines die Verbindung nit der Corticalis vermittelnden Bandes zurückzuführen. Die Veränderungen des Kerns bestehen in starker Schrumpfung und Bildung von kleinen Tropfen und grösseren Lücken, welche keine bestimmte Anordnung erkennen lassen.

Vergleichen wir diese beiden Fälle mit den bisher veröffentlichten mikroskopischen Untersuchungen von Schichtstaaren, so stellen sich einige sehr bemerkenswerthe Unterschiede heraus.

Der erste derartige Fall wurde von Deutschmann ${ }^{1}$ )

1) v. Graefe's Archiv, Bd. XXXII. 2. S. 295. 
beschrieben. Er fand in der von einem Selbstmörder herstammenden Linse einen intacten Kern, umgeben von einer doppelten Trübungszone, auf welche die im Wesentlichen intacte Corticalis folgte. Es war dies eine directe Bestätigung der bis dahin allgemein herrschenden Ansicht, dass beim Schichtstaax eine tribe Schicht zwischen dem normalen Kern und die normale Corticalis eingelagert sei.

Bald darauf konnte Beselin ${ }^{1}$ ) die Thatsache feststellen, dass der Kern zwar durchsichtig, aber in nicht geringem Grade verändert sei. Er fuihrte das Auftreten der Kernveränderungen auf eine chemische Alteration des Kerns zuruick, glaubt aber, dass die gefundenen Lüicken ein Product der Härtung seien. Als Substrat des Schichtstaars nimmt er ein System von ausgedehnten, mit feinkörnigem Detritus angefüllten Spalten an, welche den Kern fast vollständig umgeben und schon von Deutschmann beobachtet wurden.

Beselin zieht hieraus den Schluss, dass eine Ernährungsstörung zu einer chemischen Alteration der gesammten Linse führte. Die später neugebildeten Fasern konnten dem schrumpfenden Kern nicht folgen, so dass sich ausgedehnte Spalten bildeten, die als Trübungszone erschienen.

Bestätigt wurde dieser Befund und seine Deutung von Law ford ${ }^{2}$ ), welcher dieselben Veränderungen im Kern und Spalten zwischen Kern und Corticalis fand, die er für die optischen Veränderungen verantwortlich machte.

Die ausführlichste Bearbeitung erfuhr dieser Gegenstand von Schirmer ${ }^{3}$ ), welcher zunächst an der Hand von 6 Fällen den Nachweis lieferte, dass nicht die Spaltenbildung, sondern eine an der Peripherie des Kerns verlaufende Zone kleiner Lücken die Ursache der Trübung sei.

1) loc. cit.

2) Royal London ophth. Hosp. Rep., Vol. XII, p. II, S. 184.

3) v. Graefe's Archiv, Bd. XXXV, 3. S. 147; Bd. XXXVI, 1. S. 185 ; Bd. XXXVII, 4. S. 1. 
Die Kernveränderungen, welche Beselin beschrieb, konnte Schirmer ausser in seinen Fällen noch nachträglich an Deutschmann's Präparaten nachweisen. Er hält sie im Gegensatz zu Beselin für intra vitam bereits vorhandene Erscheinungen und stellt sich die Schichtstaarbildung folgendermassen vor: „Irgend eine Schädlichkeit, Ernährungsstörung unbekannter Art, ruft in den zur Zeit ihrer Einwirkung schon gebildeten wie in den während dieser Zeit entstandenen Linsenschichten Veränderungen hervor. Sie erzeugt Vacuolen, welche zwischen den Fasern liegen. Nur die jüngsten Schichten, die noch nicht zu schrumpfen begonnen hatten, die erst während der Zeit, wo die schädliche Ursache wirkte, gebildeten, enthalten eine solche Menge von Vacuolen, dass sie eine klinisch wahrnehmbare Trubung erzeugen. Nur sie bilden die Staarschicht. Ist die Anzahl dérselben im Kerne grösser, so haben wir einen stationären Kernstaar vor uns, der als Abart des Schichtstaars anzusehen ist. Der Kern schrumpft ein und die nach jener Schädigung neugebildete, aufgelagerte Corticalis kamn dem Zuge nicht folgen. A,uf diese Weise entstehen Spalten, die klinisch als sog. Reiterchen imponiren. Da ferner die Neubildung von Fasern gewiss nicht schneller vor sich geht, als unter normalen Verhältnissen, so bedingt die stärkere Schrumpfung des Kerns ein geringeres Gesammtvolumen der Linse."

Man erkennt in dieser Darstellung die Horner'sche Theorie wieder, welche annimmt, dass auf einen normalen Kern sich eine von vornherein trïbe, gebildete Schicht auflagert und nach Aufhören der Schädlichkeit wieder normale Corticalis gebildet wird. Der einzige Unterschied ist nur der, dass das Centrum des Kerns zwar verändert, aber durchsichtig ist. Während also Beselin die durch Schrumpfung des Kerns bewirkte Spaltbildung als Ursache der Schichtstaarbildung ansieht und demgemäss die Trübung erst secundär lange nach Aufhören der die Schrumpfung 
bedingenden Schädlichkeit entstehen lässt, macht Schirmer diese direct für die Entstehung der Truibung verantwortlich und sieht in der Spaltbildung nur den Ausdruck für die später eintretende Schrumpfung des Kernes, welche nicht nothwendiger Weise zu einer solchen führen muss.

Hieran reihen sich nun die von mir beschriebenen Fälle, welche die bisher bekannten Befunde zum Theil bestätigen, zum Theil aber einige Abweichungen erkennen lassen.

Zunächst besteht eine enorme Schrumpfung des Kerns, welche auch von Beselin und von Schirmer angenommen wird. Den ïberzeugenden Gründen, mit welchen Beselin an der Hand der klinischen Thatsachen und seines mikroskopischen Befundes das Vorhandensein einer solchen beweist, möchte ich noch den hinzufügen, dass der Kern in meinem Falle an der Vorder- und Hinterfläche tiefe Einkerbungen zeigt, die nicht etwa mit den sogenannten Sternstrahlen in Verbindung zu bringen sind, sondern anf eine Schrumpfung hinweisen. Dass sie nicht durch die Härtung hervorgerufen wurden, geht schon allein daraus hervor, dass sich die umgebende Flüssigkeitsschicht, welche bereits intra vitam vorhanden war, in diese Vertiefungen überall einsenkte.

Die eigenthümlichen Veränderungen des Kerns, welche bereits von den früheren Autoren beschrieben wurden, finden sich auch hier. Sie sind zweifellos schon intra vitam vorhanden, was Schirmer sowohl für seine Fälle, als auch für die früher beschriebenen nachgewiesen hat. Es handelt sich vor Allem um eine Durchsetzung der Kernsubstanz mit zahllosen kleinen Tröpfchen, welche stellenweise die Fasercontouren verdecken, an anderen Stellen spärlich auftreten. Sie sind durchaus nicht zu verwechseln mit den oben beschriebenen, durch die Messerführung an der Oberfläche entstandenen Kunstproducten, welche sich auch ausserhalb des Kerns vorfinden, während das Auftreten 
der Tröpfchen sich genau auf den Kern beschränkt. Schon aus diesem Grunde ist anzunehmen, dass sie einer specifischen Veränderung des Kerns ihre Entstehung verdanken.

Von diesen feinen Tröpfchen sind in meinem Falle scharf die wirklichen Lücken zwischen den Fasern zu unterscheiden. Sie sind von ihnen verschieden durch Grösse, Form, Inhalt, Lichtbrechungsvermögen und das Verhalten, z. B. zu Haematoxylin, und lassen keine bestimmte Anordnung erkennen, sondern liegen in spärlicher Anzahl unregelmässig in der Kernsubstanz zerstreut.

In Beselin's und in Schirmer's Fällen zeigte der Inhalt eine feinkörnige Beschaffenheit, während er hier eine durchaus homogene, in Haematoxylin schlecht färbbare Substanz darstellte. Dieser Unterschied dürfte vielleicht darin seine Erklärung finden, dass es sich in meinem Fall um einen noch nicht vor allzu langer Zeit entstandenen Schichtstaar handelte, während die übrigen untersuchten Fälle einem späteren Lebensalter angehörten - das jüngste Individuum war 5 Jahre alt - und demgemäss liegt es nahe, anzunehmen, dass der Lückeninhalt im Laufe der Zeit eine Aenderung erfährt. Im Uebrigen entspricht die Form und die Grösse genau den von Schirmer gemachten Angaben.

Der wesentliche Unterschied ron den bisher bekannten Fällen liegt nun darin, dass von einer auf dem Durchschnitt ein ovaläres Band darstellenden Zone kleinster Lïcken, welche Schirmer als das anatomische Substrat des Schichtstaars darstellt, hier nichts $\mathrm{zu}$ finden ist. Weder die Tröpfchen noch die Lücken zeigen eine Anordnung, welche mikroskopisch oder makroskopisch eine Anhäufung an der Kemperipherie erkennen liesse. Wohl sind an einzelnen Stellen die Tröpfchen an der Peripherie deutlicher erkennbar; daffïr finden sich aber auch ebenso viele, an welchen eine Ansammlung geradezu vermisst wird. Es handelt sich 
also um eine Veränderung, welche in fast gleichmässiger Weise die gesammte Kernsubstanz betroffen hat.

Ferner findet sich eine andere Erscheinung, welche in dieser Form bei Schichtstaaren noch nicht beschrieben worden ist. Es sind ausgedehnte, an der Vorder- und Hinterfläche des Kerns verlaufende Spalträume von erheblicher Breite, welche bereits intra vitam vorhanden waren und mit klarer Flïssigkeit gefüllt sind, während z. B. bei Beselin der Inhalt der Spalten sich als feinkörnig erwies. Dass es sich um eine eiweisshaltige, klare Fliissigkeit handelt, geht besonders aus der homogenen Beschaffenheit hervor, die nur durch die Messerführung beim Schneiden geringfügige Veränderungen erlitten hat. Dass in cataractösen Linsen, speciell auch in Schichtstaaren, klare Flüssigkeit enthalten ist, ist schon von $O$. Becker ${ }^{1}$ ) berichtet worden, der ebenso wie einige andere Autoren bei der Discission solcher Staare den Austritt einer solchen direct beobachten konnte.

Eine gleiche Flüssigkeitsansammlung besteht zwischen Corticalis und hinterer Kapsel, in beiden Linsen von verschieden grosser Ausdehnung, wie oben genauer ausgefübrt wurde.

Die Ansammlung sog. Morgagnischer Kugeln in diesen mit Flïssigkeit gefülten Spalträumen, wie sie in den hinteren Theilen der Linse zu Tage tritt, ist wohl, wie bereits O. Becker für andere Fälle betonte, als postmortale Erscheinung aufzufassen und man wird wohl nicht fehlgehen, wenn man die nach stattgehabter Härtung der Linsen beobachtete starke Trübung der Hinterflächen auf diese Erscheinung zurïckfuihrt.

Eigenthümlich ist ferner, dass der erkrankte Kem an keinem Punkte seiner Peripherie mit normaler Corticalis im Zusammenhang steht, sondern auch am Aequator, wo

1) 1. c. p. 159 . 
die Spaltbildung eine Unterbrechung erfahren hat, an eine stark veränderte Linsensubstanz angrenzt. Die Spalten sind zwischen Kern und Corticalis eingelagert; es erfolgte die Trennung beider Schichten genau in der Grenzlinie, während z. B. in Beselin's') Fall, wie ich mich durch Einsicht eines Präparates überzengen konnte, die Trennungslinie eine andere ist. Hier liegt an verschiedenen Stellen eine Schicht normaler Corticalis dem erkrankten Kern direct auf und erst dann folgt die Spaltbildung, während an anderen Stellen der Spalt genau die Grenze zwischen gesundem und krankem Gewebe bildet. Beselin fasst diese Unterbrechung der Spaltbildung, die besonders am Aequator sichtbar ist, so auf, dass eine doppelte Trübungszone bestehe, von denen eine an der vorderen, die andere an der Hinterfläche der Linse besser ausgebildet sei, während Schirmer grade diese Unterbrechung der Spaltbildung. am Kernäquator als Beweis gegen die Bedeutung der Spalten als Ursache der Schichtstaartrïbung anführt, indem eine solche Unterbrechung sich als Lücke geltend machen müsse, welche gerade am Aequator klinisch nicht beobachtet würde.

Diese Unterbrechung der Spalten am Kernäquator ist in meinen Fällen in ganz besonders characteristischer Weise vorhanden und ich glaube, dass diesem Befunde eine allgemeinere Bedeutung zukommt. Es findet sich auf allen Schnitten beiderseits am Kermäquator auf eine kurze Strecke beschränkt eine schon makroskopisch wahrnehmbare Trübung, die bei mikroskopischer Untersuchung sich als durch Ansammlung von Tropfen und Lücken zwischen den Linsenfasern, Veränderung des Faserinhalts und kolbige Verdickung der Faserenden bedingt erweist. Es sind dies genau

1) Durch die Liebenswürdigkeit der Herren Collegen Beselin und Schirmer, welche mir einzelne ihrer Präparate zur Einsicht überliessen, warde mir das Studium dieser Verhältnisse sehr erleichtert, wofür ich auch an dieser Stelle bestens danke. 
dieselben Veränderungen, welche die sogen. "Staarschicht" in Deutschmann's Fall darbot.

Diese intensiv getrübte Stelle vermittelt den einzigen Zusammenhang zwischen Kern und Corticalis, der sich auf allen Schnitten deutlich nachweisen lässt. Sie stellt ein circuläres, am Kernäquator verlaufendes Band dar, welches die Ernährung des Kerns allein vermittelt, ein Umstand, der meines Erachtens geradezu als ein weiterer Beweis dafür angesehen werden kann, dass der Ernährungsstrom vom Aequator aus die Linsensubstanz durchsetzt. Andernfalls müsste der Strom die Fliissigkeitsschicht durchsetzen und der geschrumpfte Kern durch Diffusion aus dieser heraus ernährt werden, eine Annahme, die mehr als unwahrscheinlich ist.

Bedingt ist diese organische Verbindung zwischen Kern und Corticalis dadurch, dass überall eine mehr oder weniger breite Schicht von Linsenfasern beiden Theilen zugleich angehört, d. h. es zieht aus dem hintern Theil der Corticalis ein Band zur vordern Seite des Kerns. Die Spaltbildung kann also keine continuirliche sein, weil die anatomische Anordnung der Fasern am Aequator eine derartige ist, dass ein Spalt, der an der Vorderseite des Kerns gelegen ist, am Aequator zwischen normale Corticalis und jenes Band ausläuft, während ein an der Hinterfläche des Kerns befindlicher Spalt am Aequator begrenzt wird vom Kern und dem zum Kern hinziehenden Bande, wie dies aus Fig. 3 ersichtlich ist.

Diese eigenthümliche Anordnung der Linsenfasern lässt sich z. B. auch in Schirmer's Fig. 10 erkennen, ebenso bei Beselin, nur liegt hier ein Band ungetribter Linsensubstanz dem Kernäquator auf, während in meinen Fällen die Linsenfasern selbst erheblich verändert sind und zwischen ihnen sich Tropfen und Lüicken vorfinden, welche eine schon makroskopisch sichtbare Trübung bedingen.

Es liegt nun sehr nahe, diese Erscheinungen darauf 
zurückzuführen, dass die enorme Schrumpfung des Kerns an jenem den Zusammenhang mit der Corticalis vermittelnden Bande die Zeichen starker Zerrung hervorgebracht hat. Dafür spricht die Lockerung der Fasern, welche zwischen sich eine erhebliche Ansammlung grosser tropfenähnlicher Gebilde und Lücken zeigen, ferner vor allem die kolbige Verdickung der Faserenden, welche in Folge der hier sich geltend machenden Zugwirkung von Seiten des Kerns in die Breite auswachsen konnten. Die Verminderung des intracapsulären Druckes führt also an dieser Stelle zu einer Verbreiterung jener Schicht, welche dem Kernäquator aufsitzt.

Die Kernschrumpfung dürfte fernerhin für einige andere Erscheinungen verantwortlich zu machen sein. Wir finden in den dem Kern benachbarten Theil der Corticalis kleine, dem Faserverlauf parallele Lücken, welche sehr wohl durch eine Zerrung des Kerns an der Corticalis zu erklären sind, welche stattfand, als beide noch in directem Zusammenhang standen. Solche kleine Lücken erwähnen Beselin und Schirmer ebenfalls; sie sind sehr wohl zu unterscheiden von jenen von $O$. Becker ${ }^{1}$ ) beschriebenen, in gesunden und cataractösen Linsen vorkommenden spindelförmigen Spalträumen.

Ferner ist- die Ablösung der hinteren Kapsel direct abhängig von der Kernschrumpfung. Wir sehen, dass jene Einkerbungen an der Hinterfläche der rechten Linse genau mit den an der Kernoberfläche sich findenden Einsenkungen correspondiren. Die elastische Kapsel vermochte dem Zuge von Seiten des schrumpfenden Kerns nicht zu folgen und so löste sie sich gerade an den Stellen $a b$, an welchen diese Zugwirkung am meisten zur Geltung kam, ein Verhalten, welches aus den Zeichnungen besser hervorgeht, als aus der besten Beschreibung.

1) Loc, cit. 
Es entsteht nun die Frage, welche Factoren wir für das Zustandekommen der intra vitam beobachteten Trübung verantwortlich machen müssen. Dass die Corticalis klar und durchscheinend sein musste, ist aus dem mikroskopischen Befund leicht erklärlich. Die zwischen Kern und Corticalis vorn und hinten angesammelte Flüssigkeitsschicht konnte eine Trübung an sich nicht bedingen, da die homogene Masse im mikroskopischen Präparat zweifellos einer intra vitam rorhandenen klaren eiweisshaltigen Flüssigkeit entspricht. Wohl aber ist es möglich, dass die Einlagerung dieser Schicht, die sich immerhin durch einen anderen Brechungsindex auszeichnet, die anderen Theile um so deutlicher hervortreten liess.

Wir müssen somit die beobachtete Tribung auf die Veränderungen des Kerns zurückführen, welche denselben in seiner ganzen Ausdehnung durchsetzen.

Die feinen punktförmigen Trübungen, welche klinisch beobachtet werden konnten, sind wohl mit grosser Wahrscheinlichkeit auf die spärlichen, im Kern zerstreuten Lücken $\mathrm{zu}$ beziehen, und so werden wir für die hauchartige Trübung der Kernsubstanz wohl jene tropfenähnlichen Einlagerungen verantwortlich machen müssen. Es genügt ja, dass nur eine minimale Differenz zwischen dem Brechungsindex jener Tröpfchen und dem der Linsenfasern vorhanden ist, um eine Trübung hervorzurufen; dabei können die Tröpfechen an sich klar und durchsichtig sein. So würde sich der geringe Grad der Trübung am ungezwungensten erklären lassen, wenn wir annehmen, dass die Tröpfchen einen nur wenig höheren Brechungsindex besitzen als ihre Umgebung.

Die intensive Trübung des ganzen Kermäquators dürfte wohl nicht nur auf Brechungserscheinungen am Rande des nur wenig getrïbten Kerns bezogen werden, sondern hier muss auch die schon an Schnittpräparaten makroskopisch v. Graefe's Archiv für Ophthalmologie. XXXIX, 1. 
sichtbare Trübung zur Geltung gekommen sein, welche dem Kernäquator aufsitzt.

Von den bisher untersuchten Fällen unterscheiden sich die meinigen also zunächst in einem sehr wesentlichen Punkt. Wir sehen, dass eine Ernährungsstörung in gleichmässiger Weise die gesammte Kernsubstanz betroffen hat, während in den anderen Fällen die Tropfen- und Lückenbildung mehr die peripheren Theile des Kerns, und nur in sehr geringem Grade die centralen ergriffen hatte. Wenn wir mit Schirmel annehmen, dass der sogen. Centralstaar nur eine Abart des Schichtstaars darstellt, bei welchem die von ihm gefundenen Veränderungen nur in stärkerem Maasse auftreten, so dürften meine Fälle wohl als solche Centralstaare aufzufassen sein, welche nur in sehr zarter Weise entwickelt sind. Dem Grad der Ernährungsstörung entspricht die hochgradige Schrumpfung des Kerns mit ihren Folge-Erscheinungen.

Wir sehen also, dass eine intra vitam als Schichtstaar angesprochene Linsentrübung sich bei der mikroskopischen Untersuchung als ein Kernstaar erweist, ebenso wie Schirmer bei der Untersuchung eines klinisch diagnosticirten Kernstaars die Merkmale des Schichtstaars fand.

Die Ansicht von Schirmer, dass das Substrat der Schichtstaartrübung in den Veränderungen des Kerns und nicht in der Spaltenbildung zu suchen ist erfährt durch meine Befunde eine weitere Bestätigung. Es finden sich hier ebensowohl Tröpfchen zwischen den Linsenfasern als auch Litcken, welche vollkommen mit den von Schirmer beschriebenen übereinstimmen und ich stimme diesem Autor darin bei, dass es sich hier um bereits intra vitam vorhandene Gebilde handelt.

Die bisherigen mikroskopischen Prüfungen von Schichtstaaren und von den ihnen genetisch nahestehenden Centralstaaren haben also als übereinstimmendes Resultat ergeben, dass eine Ernährungsstörung unbekannter Art zu 
einer spezifischen Erkrankung der Kernsubstanz führt, welche in der Bildung von eigenthümlichen Tröpfchen und Lïcken ihren Ausdruck findet.

Der Umstand, dass in meinen Fällen kein ovaläres Band kleinster Lücken vorhanden ist, würde also nach der Schirmer'schen Theorie dahin erklärt werden müssen, dass hier eine Steigerung der bei Schichtstaaren gefundenen Veränderungen vorliegt, der Art, dass die Ernährungsstörung nicht nur die perinuclearen Schichten, sondern die gesammte Kernsubstanz betroffen hat. Legt man ferner die Anschauung zu Grunde, dass sich die Corticalis erst später nach Ausbildung der Veränderungen im Kern auflagert, so wuirde es sich, genetisch betrachtet, um einen beiderseitigen Totalstaar handeln, aus welchem durch Auflagerung neuer Corticalismassen ein Kernstaar entstanden ist.

Eine derartige klinische Beobachtung beim Menschen liegt meines Wissens bis jetzt nicht vor und ich glaube, dass auch noch eine andere Möglichkeit der Entstehung jener auffälligen Veränderungen vorliegt.

Wir sehen, dass die Schrumpfung des Kerns im Stande ist, an der Grenze zwischen Kern und Corticalis Veränderungen hervorzurufen, welche zu einer Tribung führen.

Wie ich schon oben erwähnte, ist in meinen Fällen eine Zerrung der dem schrumpfenden Kern benachbarten Corticalis am Aequator in allen Schnitten direct nachweisbar, indem hier eine schon makroskopisch wahrnehmbare Tribung entstanden ist, welche durch genau dieselben Veränderungen bedingt ist, wie sie Deutschmann von der in seinem Falle gefundenen Staarschicht beschreibt. Es liegt nun sehr nahe, anzunehmen, dass die Trübung auf diesen äquatorialen Theil nur deshalb beschränkt ist, weil an den iibrigen Theilen der Kernperipherie der Zusammenhang zwischen Kern und Corticalis sich gelöst hat, so dass grosse mit Fliussigkeit gebildete Spalten entstanden. Nehmen wir an, dass die Kernschrumpfung weniger intensiv 
gewesen wäre, so ist leicht einzusehen, dass in diesem Falle im Bereiche der ganzen Kernperipherie ähnliche Erscheinungen auftreten mussten, wie wir sie hier am Aequator vor uns sehen und wie sie Deutschmann in der That beobachten konnte:

Die Spaltbildung wäre demmach ein Ausdruck für die hochgradige Schrumpfung des Kerns, wie ja auch Schirmer annimmt, und es ist desshalb durchaus nicht nothwendig, dass sie sich immer an der Grenze zwischen gesundem und krankem Gewebe findet, wie in meinen Fällen, sondern es kann ein solcher Spalt auch innerhalb der intacten Corticalis selbst auftreten, wie es z. B. in Beselin's Fall zu sehen ist. Die Spalten sind nicht continuirlich, sondern am Aequator wegen der dort bestehenden Verbindung zwischen Kern und Corticalis unterbrochen. Dieses Verbindungsstiuck wurde in meinen Fällen stark gezerrt und so kam eine circuläre Trübungszone am Kernäquator zu Stande, während in Beselin's Fall eine solche Zerrung nicht stattfand ${ }^{1}$.

Hiermit wäre die Möglichkeit bewiesen, dass die Kernschrumpfung die Ursache für eine an der Kernperipherie in mehr oder weniger grosser Ausdehnung sich findende Trubung abgeben kann. Das Material für die Trübungszone entstammt in diesen Fällen also der Corticalis und nicht dem exkrankten Kern.

1) Stellen wir uns vor, dass dieses Band in mehr oder weniger grosser Ausdehnung zerreisst, so haben wir ein Bild vor uns, wie es z. B. Otto Becker (Handbuch der gesammten Augenheilkunde v. Graefe-Saemisch Bd. V, p. 250) beschreibt. Es löst sich dann der geschrumpfte Kern und er sinkt an die tiefste Stelle der von normaler Corticalis ausgekleideten Höhle, ähnlich wie bei der Cataracta Morgagni. Ein solcher Kern wird sich bald trüben müssen, da die einzige Bahn, auf welcher Ernährungsmaterial zu ihm gelangen kann, abgeschnitten wird und das war auch in der That der Fall. 
Es wäre aber auch denkbar, dass bei geringeren Veränderungen des Kerns die Erscheinungen der Zerrung nicht im Bereiche der Corticalis, sondern an der Peripherie des erkrankten Kernes auftreten. In diesem Falle würde anzunehmen sein, dass der Zusammenhang der Corticalisschichten ein fester ist und dass die in centripetaler Richtung erfolgende Schrumpfung des Kerns zu einer Lockerung der Fasern fuilhrte, welche in einer Bildung von schmalen Lücken ihren Ausdruck fände. Diese schmalen Lüicken müssen um so mehr rundlich oder spindelförmig werden, je mehr die Schrumpfung der Fasern in der Längsrichtung zur Geltung kommt, weil dadurch eine Tendenz zur Vergrösserung der Lücken in der Breite geschaffen wird. Die Anhäufung der Lücken an der Kernperipherie wäre dann dadurch zu erklären, dass die Schrumpfung nach dem Centrum zu stattfindet, d. h. hier eine Verdichtung und an der Peripherie eine Lockerung des Gewebes eintritt. Das stimmt sehr wohl mit deu von Schirmer gefundenen Thatsachen überein.

Wenn wir somit die Lückenbildung an der Kernperipherie und im Centrum des Kerns ebenso wie die der angrenzenden Corticalis auf Zerrungserscheinungen von Seiten des schrumpfenden Kerns zurückführen können, so ist gleichzeitig leicht einzusehen, dass die Intensität der Kernschrumpfung einerseits und das Verhalten der Corticalis andererseits von wesentlichem Einfluss auf Gestalt und Grösse der Lücken ist. Ueberwiegt der Zusammenhang der Corticalisschichten gegenüber der Zugwirkung des schrumpfenden Kerns, so werden Lücken an der Peripherie entstehen. Ueberwiegt umgekehrt die Kernschrumpfung, so wird die elastische Corticalis anfangs dem Zuge nachgeben, späterhin wird es zu Zerrungserscheinungen in den an den Kern angrenzenden Schichten kommen, oder es reisst der Kern von der Corticalis in mehr oder weniger grosser Ausdehnung los und es bleibeu bandartige Brïcken am Aequa- 
tor bestehen, welche unter Umständen ihrerseits die weitere Zerrung auszuhalten haben. $Z u$ der elastischen Zugwirkung der Corticalis selbst kommt dann noch hinzu, dass die Bildung von grösseren. Spalten durch die Verkleinerung des äquatorialen Durchmessers nur begïnstigt werden kann.

Die Lückenbildung wäre demnach genetisch dasselbe wie die Spaltbildung.

Wenn wir nun beriicksichtigen, dass der Lïckeninhalt sich späterhin trübt, wie es aus den bisherigen Untersuchungen hervorgeht, und dass auch der Inhalt der Spalten eine gleiche Veränderung erfährt, wie es z. B. bei Beselin der Fall war, so wird es verständlich, dass eine doppelte Schichtstaarzone auf verschiedene Weise zu Stande kommen kann. Entweder kommt es zu Zerrungserscheinungen, welche zu Tropfen- und Lückenbildung führen oder es kommt zur Spaltbildung und es ist dann auch erklärlich, dass die zweite Zone niemals die Ausbildung und Ausdehnung erfährt, wie die ursprïngliche, da eben die Hauptwirkung der Schrumpfung sich in der Nähe der Grenze zwischen gesundem und krankem Gewebe abspielen muss. In gleicher Weise wird die klinisch beobachtete Zunahme der Schichtstaartrübung in späteren Jahren verständlich, wenn wir die Lïicken- und Spaltbildung genetisch als gleichwerthig betrachten und die Zunahme der Trübung auf eine Veränderung ihres Inhalts beziehen, welche ihrerseits eine Aenderung der optischen Eigenschaften bedingt.

Dass in meinen Fällen die Bildung einer doppelten Schichtstaarzone ausblieb, würde sich dann am einfachsten dadurch erklären, dass nach Ausbildung der grossen Spalten die Zugwirkung auf die Corticalis aufhörte und dass vorher eine Ablösung der Corticalis von der hinteren Kapsel erfolgte, welche einer zweiten Schichtstaarzone als gleichwerthig angesehen werden muss. Die Lockerung resp. Ablösung erfolgte eben hier zwischen Kapsel und Corticalis und nicht zwischen zwei Corticalisschichten. 
Es liegt ferner nahe, die Ansammlung kleinster Tröpfchen im Kern mit der Schrumpfung in Verbindung zu bringen. Wir können sehr wohl annehmen, dass bei einer Schrumpfung der einzelnen Linsenfasern der Inhalt wenigstens zum Theil ausgepresst werden muss. Es wäre dann erklärlich, warum die Tröpfchen und Fasern nahezu gleichen Brechungsindex besitzen und es wäre dann die geringe Trübung auf die Anwesenheit einer Menge kleinster Tröpfchen als solcher zu beziehen, welche an sich klar, durch stärkere Lichtbrechung eine schwache Trübung bedingen. Die Tröpfchen müssen um so kleiner ausfallen, je hochgradiger die Schrumpfung ist, und dies war auch der Fall. Eine Ansammlung solcher Tropfen an der Kernperipherie ist ebenfalls durch die Schrumpfung zu erklären, indem die in centripetaler Richtung sich einander nähernden Fasern den Inhalt in centrifugaler Richtuug auspressen müssen, und es ist desshalb leicht einzusehen, dass man wohl im Zweifel sein kann, ob man an der Kernperipherie Lücken oder Tröpfehen vor sich hat und in der That ist die Unterscheidung oft eine recht schwierige, wie auch Schirmer betont. Dass sie chemisch different sind, geht schon aus der Färbbarkeit in Haematoxylin hervor. Da wir in meinen Fällen den ganzen Kern mit Tröpfchen durchsetzt sehen und doch nur eine geringe Trübung des Kerns vorhanden war, so dürfte der Schluss berechtigt sein, dass fuir die Intensität einer Schichtstaar- oder Kernstaartrïbung die Lïcken in weit höherem Maasse verantwortlich zu machen sind, als die Tropfen.

Fine Verkleinerung des Volumens der Linse konute in meinen Fällen nicht beobachtet werden, weil diese einen Zusammenhang zwischen Kern und Corticalis voraussetzt, der hier in grosser Ausdehnung unterbrochen war. Die vorhandene Vergrösserung der Linsen erklärt sich leicht aus der postmortalen Spaltbildung.

Wenn ich somit einerseits die Ansicht Schirmer's 
durchaus theile, dass die Lücken- und Tropfenbildung im Kern das anatomische Substrat des Schichtstaars darstellt, aber andererseits mit Beselin als Ursache der Schichtstaarbildung eine Kernschrumpfung annehme, welche aber nicht nur die Spaltbildung an der Kernperipherie, sondern auch die Lücken- und Tropfenbildung im Kem hervorbringt, so bin ich mir wohl bewusst, dass noch weitere Untersuchungen nothwendig sind, um diese Frage endguiltig zu entscheiden.

Legen wir diese Anschauung zu Grunde, so ist es klar, dass alle die bei Schichtstaar gefundenen Veränderungen und Complicationen zu einer Zeit entstehen müssen, in welcher bereits wenigstens ein Theil der normalen Corticalis vorhanden war, während Schirmer annimmt, dass die Corticalis sich erst anlagert, wenn die Schädlichkeit nicht mehr wirksam ist, dass also die Lückenbildung im Kern als Ausdruck der Ernährungsstörung aufzufassen und von der später eintretenden Kernschrumpfung unabhängig ist.

Diese späte Anlagerung der Corticalis nimmt auch Beselin an. Die Kernveränderungen beträfen die ganze zur Zeit gebildetete Linse und nun lagere sich die Corticalis auf, "weil nicht einzusehen sei, wartm diese unverändert bliebe, während der gleichfalls im Anfange normale Kern von der schädlichen Einwirkung eine Áenderwng erlitt."

Als fernerer Beweis für diese spätere Anlagerung der Corticalis werden die Fälle angeführt, in welchen man erst einen Totalstaar und später einen Schichtstaar vor sich hatte. Der einzige, beim Menschen beobachtete Fall ist der von $O$. Becker ${ }^{1}$ ) mitgetheilte, wo die Entstehung einer schichtstaarartigen Trübung nach Perforation eines

1) Bericht über die Augenklinik der Wiener Universität 1863 bis 1865 . Wien 1867. 
Hornhautgeschwüres nachgewiesen wurde, während die anderen Beobachtungen sich auf Kaninchen erstrecken, bei welchen durch Discission eine Cataract erzeugt worden war. Auch hier erschien später die Corticalis wieder durchsichtig. Diese Falle sind berichtet von Leber ${ }^{1}$ ) und von Schirmer2), welch' letzterer Autor sogar die bei Schichtstaaren gefundenen perinuclearen Veränderungen constatiren konnte.

Es ist natürlich nicht zu bestreiten, dass ein Theil der Corticalis sich noch später, lange nach Ausbildung der pathologischen Veränderungen, welche den Schichtstaar bedingen, anlagern kann, jedoch bleibt die eigenthümliche Thatsache bestehen, dass ausser jenem Becker'schen Falle in welchem es sich ebenfalls um eine traumatisch entstandene Linsentrübung handelte, es noch nicht ein einziges Mal beobachtet werden konnte, dass aus einem congenitalen oder nach der Geburt entstandenen Totalstaar sich ein Schichtstaar entwickelte, und ich möchte ganz besonders hervorheben, dass die mikroskopische Untersuchung aller bisher bekannten Fälle auch nicht den geringsten Anhaltepunkt dafür liefert, dass die ganze Corticalis erst nach Beendigung des Processes, der zur Schichtstaarbildung geführt hat, gebildet wird.

Auf der anderen Seite spricht auch nicht ein einziger Grund in meinen Fällen gegen die Annahme, dass nur ein Theil der Linse erkrankte, der erkrankte Theil schrumpfte und num die bekannten Erscheinungen eintraten, welche die Praeexistenz einer Corticalschicht voraussetzen.

Der Gedanke, dass die central gelegenen Linsenfasern, in welchen die Kerne schon früh die Zeichen des physiologischen Kerntodes darbieten, eher und intensiver von einer Ernährungsstörung betroffen werden, als die besser

1) v. Graefe's Archiv Bd. XXVI, 1. S. 283.

2) Loc. cit. 
ernährten, lebensfähigen, noch mit normalen Kernen versehenen, peripheren Fasern, ist doch nicht so abschreckend, dass man ihn ohne Weiteres von der Hand weisen müsste. Die Schirmer'sche Ansicht setzt gerade das Gegentheil voraus, nämlich, dass die periphersten, jüngsten Fasern am wenigsten widerstandsfähig seien und so von der Schädlichkeit am meisten betroffen würden. Wäre dies wirklich der Fall, dann müssten doch auch in dem diese Fasern producirenden Epithel Veränderungen gefunden werden, welche auf eine vorausgegangene Erkrankung dieser Zellen schliessen liessen, aber weder in Deutschmann's noch in meinem Fall, wo die Kapsel enthalten war, waren solche Veränderungen zu finden, abgesehen von einer circumscripten vorderen Polartrübung, welche auf andere Weise zu erklären ist.

Ferner setzen die bisher geltenden Ansichten über die Entstehung des Schichtstaars voraus, dass die von keiner Seite bestrittene Kernschrumpfung erst von dem Augenblick an eintritt, wo wenigstens der grösste Theil der Corticalis angelagert ist, mit anderen Worten, dass zwischen der durch die Ernährungsstörung bewirkten chemischen Alteration der Linsensubstanz und der durch sie bedingten Schrumpfung mit ihren Folgeerscheinungen ein Zeitraum liegt, der wenigstens so gross ist, als ihn die Corticalis zur Neubildung gebraucht. Hierzu ist bekanntlich eine längere Zeit erforderlich und es ist kein Grund vorhanden, eine solche Gesetzmässigkeit anzunehmen. Es liegt daher weit näher, an eine partielle Erkrankung der Linse zu denken.

Eine weitere Stütze für diese Auffassung erblicke ich in den Messungen, über welche kürzlich $\mathrm{Dub}^{1}$ ) berichtet hat, obwohl dieser Autor dieselbe als ganz und gar unberechtigt bei Seite lässt.

1) v. Graefe's Archiv, Bd. XXXVII. 4. S. 26. 
Dub weist mit Recht darauf hin, dass seine Messungen an jugendlichen Linsen und an Schichtstaaren einen Widerspruch ergeben haben zwischen den bisherigen Anschauungen über die zeitliche und über die örtliche Entstehung des Schichtstaars und führt Folgendes aus:

Nimmt man an, die Erkrankung betreffe die peripheren Schichten der Linse, so müsste ein im ersten oder zweiten Lebensjahre entstandener Schichtstaar einen Durchmesser von wenigstens $6,8 \mathrm{~mm}$ haben, während der grösste beobachtete Schichtstaar einen solchen von $5,6 \mathrm{~mm}$ besass. Ein solcher Staar muss dann also weit früher, im Fötalleben entstanden sein. Nimmt man umgekehrt an, dass ein Schichtstaar im ersten oder zweiten Lebensjabre entsteht, und zwar in den periphersten Schichten, so müsste eine durchschnittliche Schrumpfang von $8 \mathrm{~mm}$ auf $4,4-6,6 \mathrm{~mm}$ Durchmesser eintreten, welche jedoch zu bedeutend ist, um der Wirklichlieit zu entsprechen.

Dub schliesst daraus, dass wenigstens für die kleineren Schichtstaare die Entstehung in die ersten Lebensmonate oder in die letzte Zeit des Fötallebens verlegt werden muss und lässt den Widerspruch für die grösseren Schichtstaare bestehen.

Da es nun doch wohl keinem Zweifel unterliegen kann, dass Schichtstaare ebensowohl in den ersten Lebensjahren wie im foetalen Leben entstehen können, so bedarf dieser Widerspruch der Aufklärung und dies ist nur in dem Sinne möglich, dass man eine partielle Erkrankung der Linse annimmt, die jede beliebige Ausdehnung haben kann.

Der einzige Einwand, welchen Dub gegen diese Auffassung vorbringt, ist der, dass es den natürlichen Verhältnissen vollkommen widersprechend wäre, wenn die älteren Linsenschichten, also die compacteren, widerstandsfähigeren, auf den Insult, den die Linse durch die Ernährungsstörung erleidet, reagiren, die jüngeren dagegen widerstandsfähiger sein und intact bleiben sollen. 
Dass die älteren Linsenschichten in diesem Lebensalter der Ernährungsstörung gegenüber die widerstandsfähigeren sind, bleibt noch zu beweisen. Von vornherein ist das Gegentheil wahrscheinlicher, wie ich oben ausführte. Zudem sind die älteren centralen Schichten in meinen Fällen genau so stark betroffen, wie die im Kern peripher liegenden.

Ich kamn desshalb in den Messungen von Dub nur eine weitere Stütze für die Auffassung einer partiellen Tinsenerkrankung als Ursache des Schichtstaars erblicken.

Da die Erkrankung aber nicht immer partiell zu sein braucht, so entsteht die Frage, welches Bild entstehen muss, wenn die ganze zur Zeit vorhandene Linse von der Ernährungsstörung getroffen wird. Es werden dann die Zerrungserscheinungen nur die Kapsel und ihr Epithel betreffen können, und solche Fälle sind in der That beschrieben worden.

In seiner Monographie "Zur Anatomie der gesunden und kranken Linse" (p. 149 ff.) berichtet 0 . Becker über einige Fälle von angeborenem, doppelseitigem Totalstaar, bei welchen eigenthiumliche Veränderungen in der Linsensubstanz aufgetreten waren. Zwischen den noch gut erhaltenen, Linsenfasern fanden sich kleine und kleinste Tröpfchen, welche der Abbildung und Beschreibung nach sehr an die von Schirmer beschriebenen und auch von mir gesehenen Gebilde erinnern. Es ist mir durchaus wahrscheinlich, dass hier dieselbe specifische Erkrankung der Linsensubstanz vorlag, wie beim Schichtstaar, nur fehlten hier die Lücken, und das erklärt sich leicht daraus, dass die Linse eben schrumpft, ohne dass Zerrungserscheinungen im Kern auftreten, weil hier keine Corticalschicht, sondem nu eine dünne Kapsel an die veränderte Substanz angrenzt; die Zerrungserscheinungen betreffen eben dann nur die Kapsel, resp. ihr Epithel und hier waren thatsächlich Veränderungen dieser Art zu finden. 
Durch die Schrumpfung der Linsenmassen konnten die Epithelien in die Höhe und Breite wachsen. So sehen wir in dem ersten dieser Fälle am Aequator einen epithelialen Wulst, der durch die Wucherung der äquatorialen Zellen entstehen konnte, weil die Linse nur an der vorderen Kapsel adhaerent war. Im übrigen war der Kapselsack erfüllt mit derselben homogenen Flïssigkeit, wie in meinen Fällen. In einem anderen Falle war der Kern excentrisch fixirt. Hier fehlte eine ausgedehntere Kapseltrübung, weil keine erheblichere Zerrung stattgefundeu hatte. $\mathrm{Zu}$ einer Ausbildung normaler äquatorialer Faseru ist es in keinem der vier Fälle gekommen, weil diese normalen intracapsulären Druck voraussetzt.

Becker hebt hervor, dass in diesen Beschreibungen die Genese aller derjenigen früh entstandenen Totalstaare enthalten sei, welche nicht auf entwicklungsgeschichtliche Störungen oder fötale Entzündungen zurückzuführen sind, eine Ansicht, die vielleicht nur insofern eine Einschränkung erfahren muss, als der ebenfalls von Becker beschriebeue sog. harte Kernstaar nicht hierher zu gehören scheint. Beispielsweise erklären sich dadurch jene Fälle von sog. Cataracta morgagniana bei Kindern. Sowohl der schon oben erwähnte Fall Becker's als auch die Beobachtungen von Janin und Arlt ${ }^{1}$, welche solche Staare operirten, bei denen nach Abfluss einer milchigen Flüssigkeit ein durchsichtiger Kern zurïckblieb, sind dahin zu deuten, dass die in jener specifischen Weise erkrankte Linse schrumpfte und nun von Flüssigkeit umgeben wurde, in der sie sich zu Boden senkte.

Betrifft also die specifische Erkrankung in Folge einer uns noch unbekannten Ernährungsstörung die ganze zur Zeit gebildete Linse, so entsteht ein Totalstaar, der angeboren sein oder erst später auftreten kann; betrifft sie nur einen Theil der Linse, so wird ein Central- oder Schichtstaar

1) Becker, Zur Anat. der gesunden u. kranken Linse. S. 156. 
entstehen, welcher ebenfalls der fötalen oder dex postfötalen Periode angehören und jede beliebige Grösse haben kann.

Es nähert sich somit diese Anschaunng bezüglich der Entstehung des Schichtstaars wieder mehr der alten Ansicht Arlt's, welcher ebenfalls nur eine partielle Erkrankung der Linse annahm.

Zum Schluss möchte ich noch darauf hinweisen, dass einige Complicationen, wie wir sie häufig bei Schichtstaaren beobachten, in gleicher Weise auf die Schrumpfung des Kerns bezogen werden können. So war in meinen Fällen durch Ablösung der Corticalis von der hinteren Kapsel ein mit klarer Flüssigkeit gefüllter Spaltraum entstanden, und wenn wir nun annehmen, dass der. Inhalt in späterer Zeit eine Aenderung seiner Consistenz und damit seiner optischen Eigenschaften erfährt, so ist leicht einzusehen, dass an dieser Stelle später Corticaltrïbungen entstanden sein würden. Die verschiedene Form der Spalten in meinen beiden Fällen lässt die Deutung zu, dass hier viele Variationen möglich sind, von der einfachen Polartrübung bis zur hinteren Corticalcataract. Es ist auch möglich, dass der Erkrankungsprocess zum Stillstand kommt, nachdem nur ein Spaltraum an der Hinterfläche der Linse entstanden ist, so dass der Schichtstaar nur angedeutet zu sein braucht, während ausgedehnte hintere Corticaltrübungen. bestehen. Auch ist die Ausbildung einer isolirten hinteren Schichtstaarzone, wie sie z. B. Heddaeus ${ }^{1}$ ) beschreibt, nach dem oben Gesagten leicht verständlich.

Auch liegt es nahe, die auf wenige Schnitte beschränkte Verdickung des Kapselepithels am vorderen Pol in einem meiner Fälle auf die Schrumpfung des Kerns zurïckzuführen, indem gerade an dieser Stelle der Zng besonders wirksam war, wie die Gestalt des Kerns und der im vorderen Abschnitt in Folge der starken Spannung entstan-

1) v. Graefe's Arch. f. Ophth. Bd. VIII, 1. S. 317. 
A. Peters. Ueber die Entstehung des Sehichtstaars ete. 255

dene postmortale Spaltraum beweist und man würde in gleicher Weise grössere Polar- oder Pyramidalstaare auf diese Ursache beziehen können. Auch der sog. Spindelstaar dürfte hierher gehören, bei welchem Knies ${ }^{1}$ ) eine Einkerbung der Linsenoberfläche im Bereich des vorderen Poles beobachten konnte.

Wenn ich somit fiur die Entstehung der Schicht- resp. Kernstaare und die dabei vorkommenden Complicationen einerseits und für die gewisser angeborener oder in der ersten Lebenszeit entstehender Totalstaare andererseits eine einheitliche Erklärung zu geben versucht habe, so bin ich mir wohl bewusst, dass weitere Untersuchungen über diese Frage endgültig entscheiden müssen. Insbesondere dürfte darauf $z u$ achten sein, ob die bei diesen Totalstaaren sich findenden Veränderungen der Linsensubstanz den bei Schichtstaaren beschriebenen analog sind. Dass ein solcher $\mathrm{Zu}-$ sammenhang besteht, ist bereits von $O$. Becker angedeutet worden; mangels anatomischer Untersuchungen von Schichtstaaren war es diesem leider zu früh verstorbenen Forscher jedoch damals nicht möglich, dieser Frage näher zu treten.

Erklärung der Abbildungen auf Taf. X, Fig. 1-3. Fig. 1. (Rechte Linse).
a Kern.
$b$ hintere Flüssigkeitssehicht.
$c$ Corticalis.
$d$ hintere Kapselablösung.
$e$ äquatorialer Trübungsring.
$f$ postmortal entstandener Spaltraum.
$g$ vordere Flüssigkeitsschicht.
$h$ vordere Corticalis.

Fig. 2. (Linke Linse).

$a-g$ wie Fig. 1 .

$i$ Anhäufung Morgagni'scher Kugeln.

Fig. 3. Trübungszone am Kernăquator.
a Kern.
$b$ postmortal entstandener Spalt.
$c$ gezerrte Parthie am Kernäquator.
$d$ Flüssigkeitsschicht.
$e$ Corticalis.

1) v. Graefe's Arch. f. Ophth., Bd. XXIII. 
